\title{
Projet de loi sur l'égalité salariale entre hommes et femmes
}

\section{Nicola Gundt}

\section{Q OpenEdition}

1 Journals

\section{Édition électronique}

URL : https://journals.openedition.org/rdctss/2058

DOI : $10.4000 /$ rdctss.2058

ISSN : 2262-9815

Éditeur

Centre de droit comparé du travail et de la sécurité sociale

\section{Édition imprimée}

Date de publication : 1 avril 2018

Pagination : 172-175

ISSN : 2117-4350

\section{Référence électronique}

Nicola Gundt, «Projet de loi sur l'égalité salariale entre hommes et femmes », Revue de droit comparé du travail et de la sécurité sociale [En ligne], 1 | 2018, mis en ligne le 01 novembre 2021, consulté le 13 novembre 2021. URL : http://journals.openedition.org/rdctss/2058 ; DOI : https://doi.org/10.4000/ rdctss. 2058

\section{(c) (i) (9)}

Revue de droit comparé du travail et de la sécurité sociale est mise à disposition selon les termes de la Licence Creative Commons Attribution - Pas d'Utilisation Commerciale - Pas de Modification 4.0 International. 


\section{PROJET DE LOI SUR L'ÉGALITÉ SALARIALE ENTRE HOMMES ET FEMMES}

Au cours des six derniers mois, pour une fois, le droit du travail néerlandais n'a pas connu de grands changements, car il a fallu beaucoup de temps pour se mettre d'accord sur la composition du nouveau gouvernement. Cet article portera donc sur les projets à venir plutôt que sur des réformes actuelles. Deux domaines devraient prochainement connaître des modifications: le droit du licenciement, le congé parental et le congé paternité.

\section{I - DE NOUVELLES IDÉES SUR LA LOI SUR LES LICENCIEMENTS}

La réforme de 2015 a introduit un système strict de sept motifs de licenciement. L'employeur doit pouvoir justifier pleinement un des motifs pour pouvoir licencier légalement un salarié. En outre, en cas de licenciement, l'employeur doit verser une indemnité appelée indemnité transitoire. Depuis cette réforme, les employeurs se sont plaints des sept motifs, beaucoup trop limités et demandent de pouvoir les combiner entre eux. Lors des négociations autour du nouveau gouvernement de coalition, il est apparu évident que des mesures devaient être prises sur le licenciement. Ainsi dans l'accord de coalition, figure l'acceptation des signataires de proposer une loi qui tienne compte des objections et des plaintes contre le système actuel ${ }^{1}$. En premier lieu, la future loi permettra, de nouveau, de combiner différents motifs de licenciement pour motiver un licenciement. Le principe est qu'un employeur ne peut pas et ne devrait pas continuer à employer un salarié qui ne donne pas satisfaction et qui ne peut pas s'entendre avec son employeur. Selon la loi actuelle, un de ces deux aspects doit être prouvé pour servir de motif de licenciement. Le nouveau gouvernement entend modifier cette situation. Il doit être possible de combiner les motifs si le juge estime que la relation de travail ne peut pas être poursuivie, y compris si aucun des deux motifs n'est suffisamment établi en soi. D'après le gouvernement, cet assouplissement sera compensé par une indemnité supplémentaire pouvant aller jusqu'à la moitié du montant de l'indemnité transitoire qui restera due. En substance, l'employeur aura plus de facilités pour motiver le licenciement mais devra verser au salarié une contrepartie en échange de cet assouplissement. Vu de l'extérieur, il s'agit d'une étrange décision. Après tout, soit le législateur estime qu'un licenciement fondé sur deux motifs à moitié remplis, mais se renforçant l'un l'autre, suffit à justifier un licenciement, auquel cas verser une indemnité plus élevée est un choix politique pour le moins intéressant. Soit le législateur estime que deux motifs de licenciement à moitié remplis ne correspondent pas à un motif complet, auquel cas le licenciement n'est pas un choix politique possible. Toutefois, le fait de choisir et d'accepter deux motifs à moitié remplis et une indemnité supplémentaire place cette nouvelle forme de licenciement sous le signe du compromis.

1 Regeerakkoord 2017, Para 2.1. 
L'indemnité transitoire elle-même est aussi sur le point de subir des changements s'agissant de l'indemnité à verser en cas de cessation de la relation de travail en raison d'une maladie de longue durée (plus de 104 semaines) ou en cas de licenciement pour motif économique ${ }^{2}$. L'indemnité transitoire a un double objectif: compenser la perte d'emploi et offrir des possibilités et des moyens de réinsertion et de recherche d'un nouvel emploi ${ }^{3}$. Lorsque le salarié est licencié après deux ans de maladie parce que la réintégration n'est pas possible pour l'employeur, cette compensation est considérée comme injuste. En effet, l'employeur a déjà engagé beaucoup de frais en versant au salarié son salaire pendant deux ans et aussi, probablement, en lui finançant une partie du processus de réinsertion ou de reconversion. De plus, la période d'inactivité de deux ans est également incluse dans la période durant laquelle l'indemnité est due. Par conséquent, le gouvernement a examiné différentes possibilités pour surmonter ces problèmes. La première option consistait à exempter les employeurs de l'obligation de verser l'indemnité transitoire. Mais cette option a rapidement été écartée, car, aux yeux du législateur, elle était contraire aux objectifs de l'indemnité 4 . Après tout, même une personne qui, après deux ans de maladie ou d'invalidité prolongée, perçoit une prestation d'invalidité (invalidity benefit) peut avoir la possibilité de trouver un nouvel emploi différent, auquel cas l'indemnisation pourrait s'avérer utile. Cela est particulièrement vrai pour les salariés qui ne bénéficient que de prestations d'invalidité partielle. Cependant, selon le législateur, même les salariés qui jouissent d'une prestation d'invalidité totale peuvent aussi bénéficier de l'indemnité transitoire ${ }^{5}$. En outre, l'exclusion totale du droit à une indemnité transitoire pour les salariés dans l'incapacité de travailler pendant deux ans est incompatible avec la législation néerlandaise sur l'égalité de traitement des personnes handicapées et des malades chroniques ${ }^{6}$. Selon la proposition, l'employeur devra continuer à verser l'indemnité et essayer de réinsérer le salarié, sachant que les frais engagés seront remboursés et donc compensés. L'indemnité sera versée par les caisses du chômage qui augmenteront les cotisations de 0,1\% afin de disposer des fonds nécessaires?

La proposition de réforme de l'indemnité transitoire comprend également l'idée de modifier les lois applicables en cas de licenciement économique. Selon le législateur, les lois actuelles sont considérées comme injustes et trop lourdes pour les employeurs, qui sont déjà en prise avec des difficultés économiques. Actuellement, la loi admet que des mesures équivalentes à l'indemnité transitoire, dont la valeur doit être au moins égale à l'indemnité transitoire, puissent être prévues par convention collective de travail; les conventions collectives ne peuvent donc pas stipuler des mesures visant à en réduire les coûts ${ }^{8}$. En vertu des propositions de lois, l'indemnité transitoire ne sera pas due en cas de licenciement économique si l'employeur a conclu une convention collective, avec un ou plusieurs syndicats, qui prévoit des mesures visant à prévenir ou à réduire les périodes de chômage, quelle que soit la valeur monétaire de ces mesures. L'essentiel est que les mesures conventionnelles doivent offrir de réelles perspectives de prévention ou de réduction du

2 Kamerstukken II, 2016/17, 34699, nr 3.

3 Kamerstukken II, 2013/14, 33818, nr 3.

4 Kamerstukken II, 2016/17, 34699, nr 3.

5 Ibidem.

6 Ibidem, p. 3.

7 Ibidem, p. 8.

8 Art. 7:673b BW. 
chômage ${ }^{9}$. Ces mesures peuvent être assorties d'une indemnisation financière. Si la totalité de l'indemnité doit être versée en numéraire, en général, le montant devra être égal à l'indemnité légale. Toutefois, dans le cadre de la proposition, des indemnités plus faibles sont envisageable notamment si la situation économique de l'entreprise est si grave que cela ne lui permet pas de verser ces indemnités, ou si les parties à la convention collective conviennent d'utiliser les fonds disponibles pour d'autres mesures comme les programmes de reconversion ou des mesures similaires ${ }^{10}$. À notre avis, cette proposition est périlleuse. Même si elle se limite au licenciement économique, elle semble sujette aux abus, car, par exemple, renforcer le pouvoir concurrentiel de l'entreprise peut aussi constituer un motif économique. En outre, il est intéressant de noter que le législateur opte pour une voie qui existe déjà, l'exonération par convention collective, mais qui, jusqu'à présent, n'a presque jamais été utilisée. Il existe déjà une disposition beaucoup plus facile à appliquer mais elle n'est pas largement utilisée. C'est pourquoi, à notre sens, une disposition plus compliquée, moins favorable, qui rejettera la faute sur les syndicats d'avoir détérioré les conditions de travail, n'a pas plus de chances d'être utilisée.

\section{II - L'ÉQUILIBRE ENTRE VIE PROFESSIONNELLE ET VIE PRIVÉE}

Le second domaine dans lequel des réformes sont évoquées concerne l'équilibre entre le travail et la vie privée des parents qui travaillent. Depuis 2016, le congé parental, le congé paternité et le congé de naissance ont fait l'objet de plusieurs projets d'amendements, mais récemment, une nouvelle proposition sur la prolongation du congé en cas de naissance (Wet invoering extra geboorteverlof, WIEG) a donné lieu à une consultation publique sur Internet ${ }^{11}$. La proposition du Parlement $n^{\prime}$ indique pas clairement $s^{\prime}$ il existe ou non un lien entre le socle des droits sociaux et les efforts visant à concilier vie professionnelle et vie privée. On ne trouve aucune trace d'un éventuel lien dans les «travaux préliminaires» du Conseil économique et social (Sociaal Economische Raad, SER) chargé d'émettre des recommandations.

En révisant les lois sur le congé, le Conseil souhaite atteindre trois objectifs: une plus grande participation des femmes à l'emploi, de meilleures possibilités de concilier travail rémunéré et garde d'enfants ainsi que davantage de flexibilité des modèles de temps de travail ${ }^{12}$. En ce qui concerne les besoins de l'enfant, le Conseil souligne que le congé permet d'assurer la continuité et la stabilité de la garde, toutes deux étant de grande importance. Enfin, les pères auront davantage la possibilité de s'impliquer, dès le début, dans la garde de l'enfant et les tâches ménagères, rendant ainsi la répartition plus équitable. Les possibilités de congé pendant la première année de vie de l'enfant devraient donc être élargies et les lois en vigueur (congé de naissance, congé paternel, congé parental) simplifiées ${ }^{13}$. Toutefois, les futures lois ne devraient pas entraîner de charges (financières) supplémentaires pour les employeurs. L'idée est de créer un système unifié de congé, qui ne puisse pas être transféré d'un parent à l'autre et qui corresponde au congé actuel plafonné à 26 semaines. Le ministre des Affaires sociales n'a cependant pas

9 Kamerstukken II, 2016/17, 34699, nr 3.

10 Ibidem, p. 10.

11 Concept wetsvoorstel WIEG, 19 februari 2018.

12 Recommandation du SER Optimalisering verlof na geboorte kind, advies 2018/1, 16 februari 2018.

13 Ibidem. 
retenu la proposition. Sa proposition prolonge les périodes de congé après la naissance et pendant les premières années de vie du bébé. L'objectif est de renforcer le lien entre le bébé et le parent et de permettre, dès le début, une répartition plus équitable des tâches ménagères ${ }^{14}$. En premier lieu, la proposition prolonge le congé de naissance rémunéré pour le partenaire de la mère actuellement de deux jours à l'équivalent horaire d'une semaine de travail. De plus, le partenaire a droit à cinq semaines de congé sans solde au cours des six premiers mois de la vie du bébé. Toutefois, pendant ce congé, les prestations sont accordées jusqu'à concurrence de $70 \%$ du taux de rémunération journalier maximal assuré. Le législateur entend rendre le congé du partenaire plus égal à celui de la mère, renforçant ainsi la présence de la mère sur le marché du travail ${ }^{15}$. Par ailleurs, les partenaires veulent de plus en plus participer dès le début à l'éducation de l'enfant et souhaitent répartir plus équitablement les tâches ménagères. Le fait de reléguer au second plan l'emploi rémunéré, pendant un certain temps, aide à trouver ce nouvel équilibre ${ }^{16}$. Le congé peut être pris à temps plein ou partiel, si l'employeur accepte cette dernière possibilité. En outre, les deux parents ont droit à un congé parental de 26 semaines, qui n'est toutefois pas rémunéré, à moins que l'employeur en convienne autrement.

En conclusion, le droit néerlandais n'a pas connu de grands changements, en raison de la prolongation des pourparlers de la coalition et de l'impossibilité pour le gouvernement sortant de légiférer sur des questions controversées. Cependant, les idées ont mûri entretemps et un certain nombre de propositions sont maintenant à l'agenda dans les Chambres du Parlement. Les deux réformes analysées ci-dessus auront, si elles sont adoptées, des conséquences sur l'avenir du droit du travail néerlandais, car elles touchent à la rupture des contrats de travail et à la manière de trouver un équilibre entre l'intérêt de l'employeur de disposer facilement d'une main-d'œuvre et l'intérêt des salariés d'avoir du temps en cas de changements importants et décisifs dans leur vie privée. En ce sens, les changements s'inscrivent dans la lignée - et dans le cas du licenciement, partiellement à contrecourant des réformes des deux dernières années, à commencer par la loi sur l'emploi et la sécurité ainsi que la loi sur le travail sans limite de temps et de lieu.

14 Cela été déjà prévu dans les précédentes ébauches, Kamerstukken II, 2016/17, 34617, nr. 3 p. 1.

15 Concept wetsvoorstel WIEG, 19 februari 7.

16 Ibidem, p. 8. 